\title{
Spin Multiplicity and Solid-State Electrochemical Behavior in Charge-Transfer Co-crystals of DBTTF/F4TCNQ
}

\author{
Federica Solano, Paolo Inaudi, Mario Chiesa, Gabriele Kociok-Köhn, Enrico Salvadori, \\ Enrico Da Como,* Davide Vanossi, Mery Malandrino, Raanan Carmieli, Agnese Giacomino,* \\ and Claudio Fontanesi
}

Cite This: J. Phys. Chem. C 2021, 125, 8677-8683

Read Online

ACCESS | Lill Metrics \& More | 回 Article Recommendations | si Supporting Information

ABSTRACT: Charge-transfer crystals exhibit unique electronic and magnetic properties with interesting applications. The chargetransfer single crystal formed by dibenzotetrathiafulvalene (DBTTF) together with 2,3,5,6-tetrafluoro-7,7,8,8-tetracyanoquinodimethane (F4TCNQ) presents a long-range ordered supramolecular structure of segregated stacks, with a unitary degree of charge transfer. Thus, the crystal structure is composed of dimerized radical molecules with unpaired electrons. The energy levels and the spin degrees of freedom of this material were investigated by solid-state electrochemistry and electron paramagnetic resonance (EPR) spectroscopy. The electrochemical
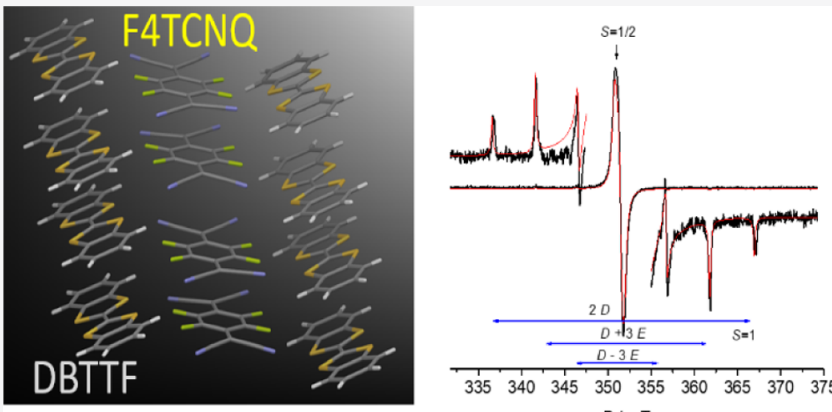
data, supported by density functional theory calculations, show how this organic Mott insulator has an electronic gap in the range of hundreds of meV. EPR experiments show the presence of a ground-state $S=1$ triplet spin state along with localized $S=1 / 2$ spins. The calculations also predict a ground-state triplet configuration, with the singlet configuration at $170 \mathrm{meV}$ higher energy. DBTTF/F4TCNQ seems to be a candidate material for organic electronic and spintronic applications.

\section{INTRODUCTION}

Charge-transfer complexes arise from the combination of two neutral molecules with different electron affinities, which, by mutual polarization, induce the transfer of an electron from a donor (D) to an acceptor (A), commonly referred to as a D-A pair. ${ }^{1}$ The charge-transfer interaction is crucial in the solid state and leads to the formation of some typical crystal structures, where the donor and acceptor molecules stack with their $\pi$-conjugated orbitals facing each other in an alternating fashion. ${ }^{2,3}$ Another common solid-state arrangement is when the donor and the acceptor form parallel stacks with only a $\pi$ overlap between molecules having the same molecular structure, that is, only donors or only acceptors. ${ }^{4}$ These are referred to as segregated stack charge-transfer co-crystals. Recently, charge-transfer co-crystals have been used to demonstrate new interesting applications. ${ }^{1}$

In the vast literature on these systems, one of the first organic metals, tetrathiafulvalene/2,3,5,6-tetrafluoro-7,7,8,8 tetracyanoquinodimethane (TTF/TCNQ), has emerged as a prototype system and played a central role in the development of the field of organic electronics. ${ }^{5}$ In this case, the TTF and TCNQ molecules stack into segregated stacks with a charge transfer that does not correspond to a complete unit of charge. This is a prerequisite for metallic behavior where a partially filled electronic band contributes to conduction. ${ }^{6}$ When complete charge transfer occurs, that is, when both the donor and acceptor exchange a complete unit of charge, an ionic crystal is formed by the cation of the donor and the anion of the acceptor. ${ }^{7,8}$ Such systems have not received equally extensive attention in the literature, mainly because their performances as conductors were observed to be poor. ${ }^{9}$ In fact, the presence of ionized molecules in the lattice increases the on-site charge repulsion when electrons have to delocalize or move in response to an external field. This gives rise to what is commonly known as a Mott insulator or Mott gap behavior in electrical transport. ${ }^{10}$ The unpaired electrons, on these molecular ions, within the crystal lattice open up interesting possibilities when looking at the spin degrees of freedom. This aspect has been recently recognized by Wudl and colleagues. ${ }^{11}$ Thus, charge-transfer co-crystals combine the high conductivity or semiconductor behavior together with the unpaired or paired (singlet or triplet multiplicity) spins. The charge and

Received: January 2, 2021

Revised: April 1, 2021

Published: April 20, 2021 
spin degrees of freedom could be combined toward the design of new organic multiferroic materials. ${ }^{12,13}$

In this paper, a charge-transfer co-crystal with peculiar electronic and magnetic properties based on DBTTF/ F4TCNQ is investigated. A detailed structural characterization by single-crystal X-ray diffraction reveals that the material unit cell is composed of homodimers of DBTTF and F4TCNQ which are arranged in segregated stacks. The spin degrees of freedom were investigated using electron paramagnetic resonance (EPR) spectroscopy, which show the coexistence of spin doublet $(S=1 / 2)$ and triplet $(S=1)$ states at room temperature. The material is an electroactive Mott insulator with well-defined frontier orbitals capable of accepting electrons and holes, as demonstrated by the solid-state electrochemistry data. These data were modeled using a density functional theory (DFT) cluster approach, which captures the EPR observations and is able to describe the novel electrochemistry data. Typically, electrochemical-based cyclic voltammetry $(\mathrm{CV})$ measurements are performed for studies in solution; here, to probe the crystal directly and avoid the dissolution of the solid-state material in solution, a new custom-designed setup was adopted. The approach is based on a solid-state electrochemical configuration fully described in the Supporting Information. ${ }^{14-16}$ The energies of the highest occupied molecular orbital (HOMO) and the lowest unoccupied molecular orbital (LUMO) levels and of the related band gap, $E_{\mathrm{BG}}$, were determined from the electrochemical results. EPR experiments reveal a rather complex picture for the electronic structure, where localized doublet radicals on DBTTF coexist along with the triplet species, found at a much lower concentration. Ab initio DFT calculations (CAMB3LYP/cc-pVTZ) were used to rationalize the experimental evidence in terms of a molecular perspective.

\section{EXPERIMENTAL AND THEORETICAL METHODS}

Chemicals. DBTTF (99\% purity) was purchased from Sigma-Aldrich, and 2,3,5,6-tetrafluoro-7,7,8,8-tetracyanoquinodimethane ( $98 \%$ purity) was purchased from TCI, UK. The materials were purified using a vacuum sublimation apparatus. Acetonitrile anhydrous, ACN ( $<99 \%$ purity), tetrabutylammonium hexafluorophosphate, TBAPF6 (99\% purity), and 5\% Nafion 117 solution were obtained from Sigma-Aldrich and were used for electrochemical studies. During all electrochemical analyses, alumina powder was used to renew the electrode surface. All glassware were cleaned by high-purity water (Millipore $>20 \mathrm{M} \Omega \mathrm{cm}$ ).

Electrochemical Setup. The electrochemical analysis was performed using a PalmSens 3 portable potentiostat (PalmSens, Houten, Netherlands). It was interfaced to a tablet or laptop computer, and the software PSTrace 4.6 was used to set the process parameters. A conventional three-electrode cell was used for electrochemical studies of pristine solutions. The working electrode (WE) selected was a glassy carbon electrode (GCE) with a diameter of $3 \mathrm{~mm}$. The counter electrode (CE) was a Pt electrode. The potentials were measured against a $\mathrm{Ag} /$ $\mathrm{AgCl} / \mathrm{KCl}_{\text {sat }}$ reference electrode (RE). A peculiar arrangement was adopted in the case of co-crystals to preserve their solid state: a cylindrical Teflon cell featuring a hole $(0.8 \mathrm{~cm}$ diameter) in the bottom was used in a vertical configuration, where a glassy carbon plate (GCP) (size: $25 \mathrm{~mm} \times 25 \mathrm{~mm}$ ) was tightened from below; a Teflon ring was used to ensure no solution leakage from the cell. The cell was rested/leaning on a copper plate as the electrical contact. The GCP was used as
WE, while a Pt wire and $\mathrm{Ag} / \mathrm{AgCl} / \mathrm{KCl}_{\text {sat }}$ were used as $\mathrm{CE}$ and $\mathrm{RE}$, respectively. $\mathrm{CE}$ and $\mathrm{RE}$ were placed at the top of the cylinder in contact with the electrolytic solution. The cocrystals were placed over the WE and covered with a Nafion membrane. Then, the membrane was left "to dry" for $5 \mathrm{~min}$, and the electrolytic solution was added. The Nafion membrane prevents direct contact between the solid co-crystal and the electrolytic solution, avoiding the dissolution of the co-crystals but preserving the ion transfer. In fact, if dissolved in solution, the co-crystals would be divided, giving the individual species.

Charge-Transfer Co-crystal Preparation and X-ray Structure. DBTTF/F4TCNQ co-crystals were grown using a horizontal quartz tube under an argon stream. This growth method is widely used for organic materials ${ }^{17}$ and recently has been employed by our group and others to grow high-quality organic co-crystals. ${ }^{8,18,19}$ Argon gas was obtained from BOC, $\mathrm{UK}$, with a purity of $6 \mathrm{~N}$. The tube was filled with quartz tubes of smaller diameter and $10 \mathrm{~cm}$ length, used to harvest the crystals at the end of the growth procedure. The tube, with 150 $\mathrm{mg}$ of each material in powder form, was placed in a Carbolite EHC 12/600B three-zone furnace, with the hot zone at $507 \mathrm{~K}$, the center of the furnace at $466 \mathrm{~K}$, and the cold zone at $420 \mathrm{~K}$ for the co-crystal DBTTF/F4TCNQ. The tubes were cleaned by ultrasonication in deionized water, 2-propanol, and acetone, sequentially. For structure determination, a single crystal was mounted on a Dual-Thickness MicroMount. Intensity data were collected on a Rigaku Supernova Dual, EosS2 system using monochromated $\mathrm{Cu} \mathrm{K} \alpha$ radiation $(\lambda=1.54184 \AA)$. Unit cell determination, data collection, and data reduction were performed using the CrysAlisPro software. The structure was solved with SHELXT and refined by a full-matrix least-squares procedure based on F2(SHELXL-2018/3). ${ }^{20}$ All non-hydrogen atoms were refined anisotropically. Hydrogen atoms were placed onto calculated positions and refined using a riding model.

EPR Spectroscopy. Room-temperature X-band CW EPR spectra were collected with a super-high Q resonator (ER4122 SHQE, operating at $\sim 9.8 \mathrm{GHz}$ ). Low-temperature Q-band CW EPR spectra were collected using the EN 5107D2 Bruker resonator housed in an Oxford CF935 cryostat. Detailed experimental conditions are reported in the figure captions. All samples were finely ground in order to obtain a powder averaged spectrum. Spectra simulations were performed using EasySpin 5.2.28 toolbox $^{17}$ within the Matlab $2019 \mathrm{~b}$ software suite (The Mathworks Inc., Natick, MA).

Calculation Details. Theoretical calculations have been performed within the framework of ab initio linear combination of atomic orbitals using the FireFly, GAMESS, and ORCA suite of programs. Concerning the DBTTF and F4TCNQ single-molecule constituents, calculations were performed at both the CAMB3LYP/6-31G(d) and CAM$\mathrm{B} 3 \mathrm{LYP} / \mathrm{cc}-\mathrm{pVTZ}$ levels of theory for the sake of comparison to evaluate the reliability of $6-31 \mathrm{G}(\mathrm{d})$. DFT calculations concerning the crystal were performed by the extraction of a "cluster" of molecules, maintaining the experimental geometrical disposition exactly as obtained by experimental X-ray diffraction data analysis. In the case of the DBTTF/F4TCNQ co-crystal, the data reported in the following are obtained by considering explicitly a two (DBTTF)-by-two (F4TCNQ) cluster. 


\section{RESULTS AND DISCUSSION}

Figure 1 shows the molecular structures and unit cell of DBTTF/F4TCNQ in different orientations. This material has (a)

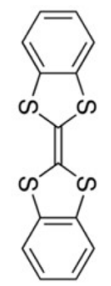

(b)

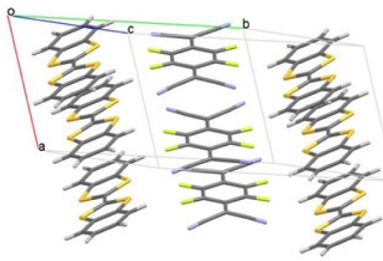

(d)

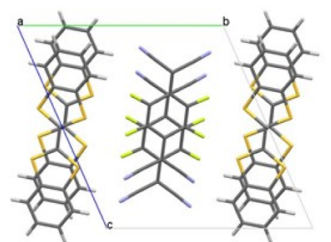<smiles>N#CC(=N)c1c(F)c(F)c(C(C#N)=C(C#N)C#N)c(F)c1F</smiles>

(c)

(e)
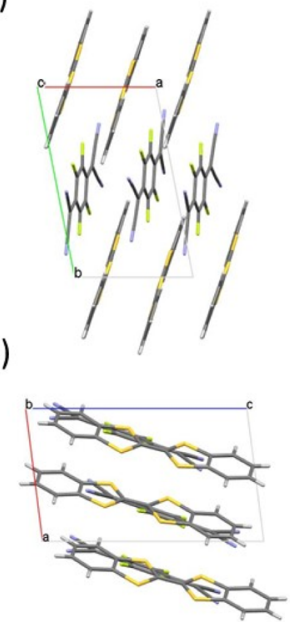

Figure 1. (a) Molecular structures of DBTTF and F4TCNQ. (b) Crystalline structure of DBTTF/F4TCNQ co-crystals, here showing a total of nine molecules with unit cell axes; the number of molecules per unit cell is four. The unit cell axes are labeled. (c-e) Projections of the unit cell along the $c, a$, and $b$ unit cell axes. Unit cell axes at 170 $\mathrm{K}$ are $a=7.4984(2) \AA, b=13.0612(5) \AA$, and $c=13.6469(6) \AA$.

a triclinic unit cell with the space group $P \overline{1}$, and the stoichiometry of the co-crystal is $1: 1$. Our structure compares well with the first X-ray study on this system by Emge et al. ${ }^{20}$ Two peculiar features are the segregated stack of donors and acceptors and the dimerization of both DBTTF and F4TCNQ. The dimerization is clearly apparent in the F4TCNQ stack, as can be seen in Figure 1a,b. At $170 \mathrm{~K}$, the interplanar distances between F4TCNQ molecules are 3.223 and $3.416 \AA$, whereas those between DBTTF molecules are 3.418 and $3.491 \AA$. At room temperature $(300 \mathrm{~K})$, the dimerization persists, and the distances become 3.281 and $3.456 \AA$ for F4TCNQ and 3.477 and $3.526 \AA$ for DBTTF. Therefore, F4TCNQ molecular planes are not only closer to each other on average but also show a larger degree of dimerization, both at $170 \mathrm{~K}$ and room temperature.

The crystal symmetry was investigated in a range of temperatures from $80 \mathrm{~K}$ to room temperature. The crystal remains triclinic with the space group $P \overline{1}$. A detailed analysis of the single-crystal structures at different temperatures will be reported elsewhere.

The EPR powder spectra of the co-crystal were recorded at room temperature at $\mathrm{X}$-band frequency and are shown in Figure 2. The spectrum of DBTTF/F4TCNQ is characterized by a relatively intense absorption line at $g \approx 2.00$ (magnetic field $\approx 352 \mathrm{mT}$ ), assigned to $S=1 / 2$ radical species. Lowtemperature Q-band experiments allow resolving the aniso-

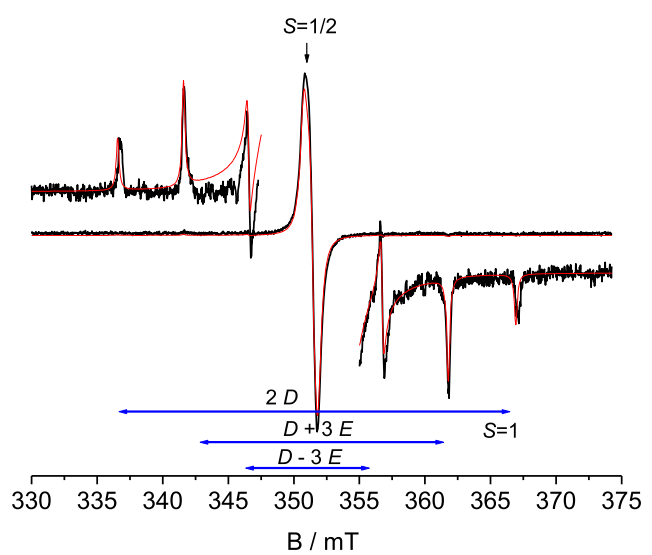

Figure 2. Room-temperature experimental (black curve) and simulated (red curve) CW X-band EPR spectra of DBTTF/ F4TCNQ co-crystals in the powder form. The spectral features belonging to the triplet state $(S=1)$ in the spectrum are indicated by the blue double-sided arrows. The spectra were recorded at $13 \mathrm{~dB}$ attenuation and a modulation amplitude of $0.1 \mathrm{mT}$. The parameters used in the simulation with EasySpin are reported in the Supporting Information.

tropic $\mathbf{g}$ tensor (Supporting Information, Figure S1), which agrees well with the literature values for the DBTTF cation ${ }^{21}$ and with $g$-factors obtained for this radical from DFT calculations (Supporting Information Table S1). Based on these observations, the recorded doublet state can thus be assigned to isolated radical species, possibly defects, or highly localized spin $1 / 2$ on DBTTF. Preliminary measurements on a single crystal reassure that the radical is not induced by grinding, in keeping with similar observation reported in the case of 5-alkylphenazinium-TCNQ charge-transfer crystals. ${ }^{22}$ Superimposed to the narrow doublet signal, a weaker six-line pattern is observed, which can be safely attributed to $S=1$ species. A convincing simulation of this signal (red curve in Figure 2) was obtained using the spin-Hamiltonian

$$
\hat{\mathcal{H}}=\mu_{\mathrm{B}} S \mathbf{g} B+D\left(S_{z}{ }^{2}-\frac{2}{3}\right)+E\left(S_{x}^{2}-S_{y}^{2}\right)
$$

and assuming an isotropic $g$ value $(g=2.0038)$ and the zerofield splitting parameters $|D|=426 \mathrm{MHz}$ and $|E|=46.8 \mathrm{MHz}$. These values are very similar to those obtained for the triplet ground state observed for 5-alkylphenazinium-F4TCNQ ion pairs. ${ }^{23}$

Figure 3a shows the $\mathrm{CV}$ curve recorded for pristine F4TCNQ in AN solution, using tetrabutylammonium hexafluorophosphate (TBAPF6) as the supporting electrolyte and GCE as WE, at $50 \mathrm{mV} \mathrm{s}^{-1}$ (other scan rates are reported in the Supporting Information). The CV curve of F4TCNQ is in good agreement with the data already reported in the literature. ${ }^{24}$ Two reduction/oxidation peaks are present in the forward-negative/backward-positive potential scan direction; they are of reversible nature with a peak-to-peak separation of about $60 \mathrm{mV}$. As F4TCNQ is an electron acceptor, the reduction peaks, $E_{\mathrm{PC}}$, are particularly interesting. These are found at 0.543 and $0.062 \mathrm{~V}$ and correspond to the formation of the anion F4TCNQ ${ }^{(-)}$and dianion F4TCNQ ${ }^{(2-)}$, respectively, as indicated in Figure 3a. It was observed that F4TCNQ is characterized by chemical stability issues, likely related to the reactivity of the cyano groups with humidity and water traces in the ACN solutions. ${ }^{24}$ A systematic study on 


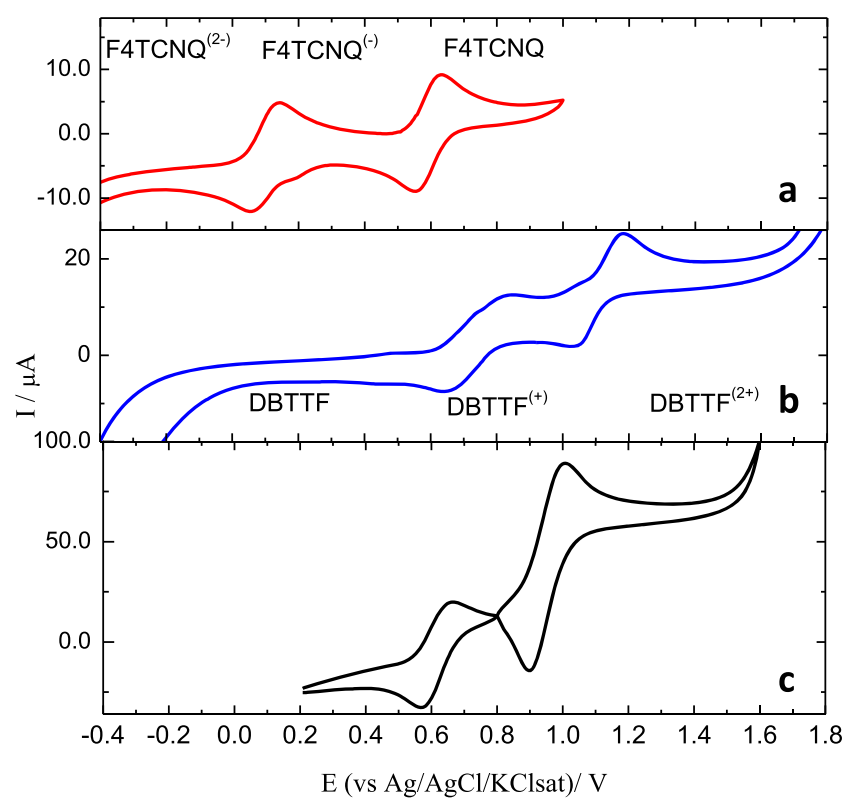

Figure 3. $\mathrm{CV}$ curves of the pristine organic compounds in $0.1 \mathrm{M}$ TBAPF6 ACN solution containing (a) $1 \mathrm{mM}$ F4TCNQ and (b) DBTTF powder with Nafion. (c) CV curve of DBTTF/F4TCNQ cocrystals. WE/GCE for (a) and GCP for $(\mathrm{b}, \mathrm{c}), \mathrm{RE}: \mathrm{Ag} / \mathrm{AgCl} / \mathrm{KCl}$ sat, $\mathrm{CE}$ : Pt wire. CV profiles were obtained at $50 \mathrm{mV} \mathrm{s}^{-1}$ scan rate.

F4TCNQ stability as a function of time in the adopted experimental conditions was carried out (details are reported in the Supporting Information), and it allowed to ensure a total reliability of our electrochemical measurements.

Figure $3 \mathrm{~b}$ shows the DBTTF solid-state CV performed on the powdered material. To the best of our knowledge, the DBTTF solid-state CVs have not yet been reported in the literature. Two clear oxidation peaks appear in the forward oxidation scan: the relevant underlying redox processes appear reversible, with a peak-to-peak separation of about $100 \mathrm{mV}$. The anodic peaks, $E_{\mathrm{PA}}$ are 0.85 and $1.183 \mathrm{~V}$ and correspond to the formation of the DBTTF mono- and dications. These anodic peaks are at a higher voltage compared to those recorded in solution (Supporting Information, Figure S3), pointing to the solid-state effect in the shift of HOMO levels. It is important to note that the redox potentials for F4TCNQ/ $\mathrm{F}_{4 T C N Q}{ }^{(-)}$and $\mathrm{DBTTF} / \mathrm{DBTTF}^{(+)}$are very close. If we consider that in the solid state, the F4TCNQ LUMO is predicted to shift by up to $1.3 \mathrm{eV}$ with respect to the vacuum level, ${ }^{25}$ the domain of electrical potentials corresponding to the stability of F4TCNQ ${ }^{(-)}$overlaps with that of the DBTTF monocation. Thus, the electrochemistry of the pure compounds suggests a favorable charge-transfer reaction and the formation of a co-crystal with integer charge transfer, in accordance to the analysis of the charge-transfer degree by IR spectroscopy. $^{25}$

Figure 3c shows the CV profiles of solid DBTTF/F4TCNQ co-crystals, using GCP as WE, after covering it with the Nafion membrane (details on the solid-state CV method are provided in the Supporting Information). Panel (c) reports two CV curves, which are recorded starting from the open-circuit potential and are separated for the anodic and cathodic scans. The data show two quasi-reversible redox processes (two current peaks are evident in both the cathodic and anodic scans; moreover, they are, within each scan, almost of the same current intensity). It is possible to observe that the CV curve of the cocrystal (i) does not correspond to the sum of the CV curves of the individual compounds, (ii) is characterized by two redox processes occurring in the voltage ranges $0.5-0.7$ and $0.9-1.1 \mathrm{~V}$, and (iii) is characterized by larger current in the oxidation scan. As the co-crystal is characterized by a high degree of charge transfer, DB is considered as a cation and F4 as an anion, which means $\left(\mathrm{DBTTF}^{1+} / \mathrm{F}_{\mathrm{TCNQ}} \mathrm{TCN}^{1-}\right)$. This is supported by EPR and the arguments presented above on the CV curves of the single materials. The peak at about $+1.0 \mathrm{~V}$ is assigned to the oxidation of DBTTF molecule to its dication $\left(\mathrm{DBTTF}^{(+)} / \mathrm{F}_{4} \mathrm{TCNQ}^{(-)}\right.$oxidation to $\left.\mathrm{DBTTF}^{2+} / \mathrm{F}^{\mathrm{TCNQ}(-)}\right)$. An almost symmetrical reduction peak is found in the backward curve at about $+0.90 \mathrm{~V}$, which is assigned to the relevant quasi-reversible reduction process. In the cathodic $\mathrm{CV}$ scan, the reduction peak centered at about $+0.57 \mathrm{~V}$ corresponds to the reduction of the acceptor or the donor on the surface of the crystal, giving respectively DBTTF/ F4TCNQ $^{(-)}$or $\mathrm{DBTTF}^{(+)} / \mathrm{F}_{4} \mathrm{TCNQ}^{(2-)}$.

Remarkably, the peak-to-peak current difference in the cathodic CV scan is about $60 \%$ smaller than the peak-to-peak current difference in the anodic one. This result suggests that the oxidation is more efficient than the two processes in reduction: the larger current can be due to a more effective ability of the DBTTF stack to exchange its spin $1 / 2$ uncorrelated electrons. If, instead, the oxidation involves the F4TCNQ stacks, their close dimerization distance, cf. Figure 1,

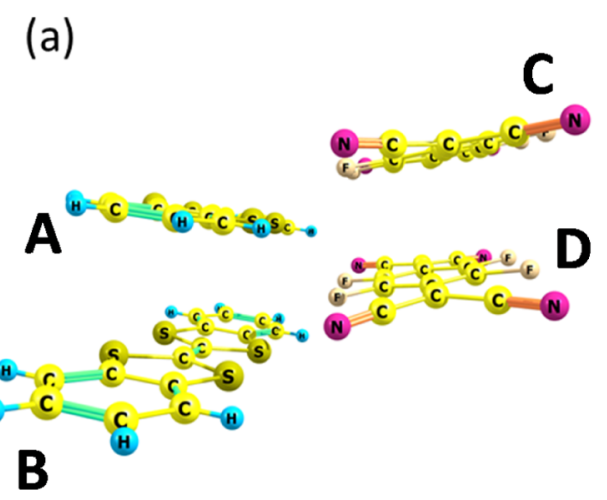

(b)

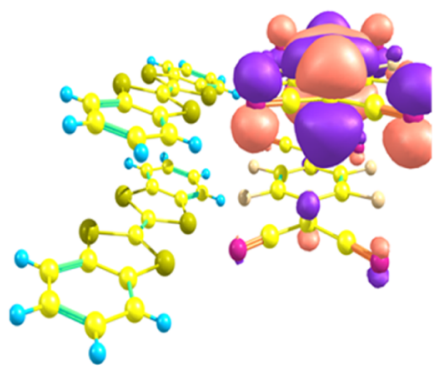

(c)

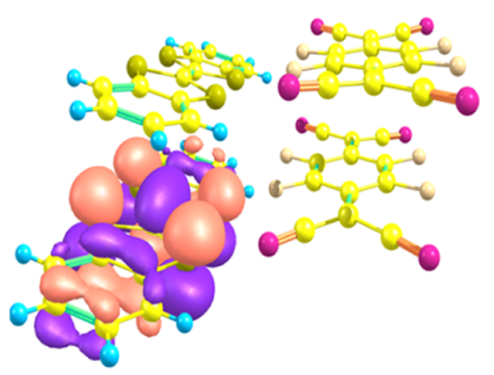

Figure 4. Graphical representation of the DBTTF/F4TCNQ $2 \times 2$ superstructures used in the theoretical calculations; atomic coordinates were obtained from the relevant experimental, X-ray, and co-crystal geometries of Figure 1. (a) Labeling of molecules in the cluster. (b) Calculated HOMO and (c) LUMO. 
Table 1. DBTTF/F4TCNQ Cluster: LUMO, HOMO, Mulliken Net Charge, and Energy Stability Values for the Singlet, Triplet, and Quintet Electronic State Multiplicity ${ }^{a}$

\begin{tabular}{|c|c|c|c|}
\hline & $\begin{array}{l}\text { singlet } \\
\text { vacuum }\end{array}$ & $\begin{array}{c}\text { triplet } \\
\text { vacuum }\end{array}$ & $\begin{array}{l}\text { quintet } \\
\text { vacuum }\end{array}$ \\
\hline LUMO & -4.622162376 & -4.65182302 & -4.664019096 \\
\hline HOMO & -5.90355662 & -5.284764836 & -5.000074301 \\
\hline HOMO/LUMO gap & 1.281394244 & 0.632941816 & 0.336055205 \\
\hline charge DBTTF(A) & 0.31376 & 0.911646 & 0.912747 \\
\hline charge DBTTF(B) & 0.474524 & 0.873542 & 0.875194 \\
\hline charge F4TCNQ(C) & -0.44138 & -0.90325 & -0.85547 \\
\hline charge F4TCNQ(D) & -0.34691 & -0.88194 & -0.93247 \\
\hline energy tot & -6411.10742583 & -6411.113703 & -6411.106642 \\
\hline $\mathrm{kcal} \mathrm{mol}^{-1}$ & 3.939300805 & 0.0 & 4.431201 \\
\hline$S^{* * 2} 2$ & & 2.8511 & 6.1011 \\
\hline
\end{tabular}

could help charge delocalization. To help these assignments, quantum chemistry calculations were performed.

The theoretical results are presented here, focusing on the (i) band gap and frontier orbital energy variation of DBTTF and F4TCNQ as isolated molecules compared to the values of structures from the co-crystal, (ii) charge distribution within the donor-acceptor partners in the co-crystal, and (iii) the spin multiplicity of the energy levels for the superstructure representative for the co-crystal. For the co-crystal, theoretical calculations were carried out using a cluster strategy. The cluster shown in Figure $4 \mathrm{a}$ was considered, which is representative of one unit cell with a total of four molecules: two DBTTF and two F4TCNQ. In both cases, the geometry of the cluster was maintained fixed, according to the experimental X-ray data (see Figure 1).

Table 1 summarizes the theoretical values of HOMO, LUMO, the atomic Mulliken net charge, as well as the total molecular electronic energy. These physical quantities are reported for different values of the total spin in the cluster, that is, a singlet, triplet, or quintet spin state. The charge is factorized for each molecule forming the cluster; thus, the considered unit cell is neutral. Four molecules are labeled according to the scheme in Figure 4a. The degree of intermolecular charge transfer can be made quantitative, exploiting the Mulliken electron density decomposition scheme. Interestingly, a complete single-electron transfer occurs only in the triplet and quintet: -1.8 electrons is the net charge on two F4TCNQ molecules for the triplet and also quintet. The degree of charge transfer in the singlet state is smaller by about 50\%, and one unit of charge is shared between two F4TCNQ acceptor molecules, which is inconsistent with the experimental evidence. ${ }^{25} \mathrm{~A}$ comparison of the total electronic energies of the singlet, triplet, and quintet states shows that the triplet is the most stable. The singlet and quintet are just 16.32 and $18.41 \mathrm{~kJ} \mathrm{~mol}^{-1}$ higher in energy, respectively. Thus, the theoretical results suggest a triplet ground state but with the singlet and quintet states that can be thermally populated.

Figure $4 b, c$ shows the frontier molecular orbitals. It is possible to observe that the HOMO is localized on the F4TCNQ molecules, while the LUMO is on the DBTTF. This result is also consistent with the Mulliken net charge variation during simulated oxidation and reduction processes.

Table 2 shows that oxidation leads to a prominent decrease in the negative F4TCNQ charge, which is left almost neutral. In the reduction process, a larger variation is found for
Table 2. DBTTF/F4TCNQ $2 \times 2$ Cluster, CIF300K UCAM-B3LYP/6-31G*

\begin{tabular}{cccccc} 
& neutral & anion & $\begin{array}{c}\text { variation } \\
0 \rightarrow \text { anion }\end{array}$ & cation & $\begin{array}{c}\text { variation } \\
0 \rightarrow \text { cation }\end{array}$ \\
$\begin{array}{c}\text { charge } \\
\text { DB 1 }\end{array}$ & 0.911646 & -0.0491 & -0.9607 & 0.3477 & -0.56395 \\
$\begin{array}{c}\text { charge } \\
\text { DB 2 }\end{array}$ & 0.873542 & -0.0768 & -0.9503 & 0.6498 & -0.22374 \\
$\begin{array}{c}\text { charge } \\
\text { DB } \\
\text { both }\end{array}$ & 1.785188 & -0.1259 & -1.9111 & 0.8781 & -0.90709 \\
charge \\
$\begin{array}{c}\text { F4 } \\
\text { both }\end{array}$ & -1.785188 & -0.8740 & 0.91119 & 0.1218 & 1.90699 \\
charge \\
$\begin{array}{c}\text { F4 } 1^{\text {st }} \\
\text { charge }\end{array}$ & -0.90325 & -0.4659 & 0.43735 & 0.0830 & 0.98625 \\
$\begin{array}{l}\text { F4 2 } \\
\text { nd }\end{array}$ & -0.88194 & -0.4080 & 0.47394 & 0.0388 & 0.92074 \\
\hline
\end{tabular}

DBTTF, which results almost neutral in the reduced crystal. This analysis helps in assigning the experimentally determined redox processes of Figure 3c, where the reduction is likely to result in $\mathrm{DBTTF} / \mathrm{F} 4 \mathrm{TCNQ}{ }^{(-)}$and the oxidation peak in the formation of $\mathrm{DBTTF}^{(+)} / \mathrm{F} 4 \mathrm{TCNQ}$.

\section{CONCLUSIONS}

The comparison of data obtained by CV, EPR, and X-ray techniques, supported by theoretical DFT-based calculations, allowed us to obtain a clear picture of the electronic and spin phenomena occurring in the charge-transfer co-crystal formed by DBTTF and F4TCNQ at room temperature.

The degree of charge transfer in DBTTF/F4TCNQ is close to a single unit of charge, which means that the molecular units have unpaired spins in their frontier orbitals. The EPR results give a clear indication of the existence of a dominant DBTTF radical cation species in the solid state, together with a triplet signal due to spin-spin interactions between charges likely on the strongly dimerized F4TCNQ stacks. The solid-state electrochemistry data show reversible cycles, which, on the basis of the theoretical results, can be ascribed to the reduction of $\mathrm{DBTTF}^{(+)}$in the co-crystal to neutral DBTTF and oxidation of F4TCNQ ${ }^{(-)}$to neutral F4TCNQ on the anodic scan. The overall experimental picture is consistent with the theoretical results, which show that for a $2 \times 2$ cluster the triplet appears to be the most stable electronic state, and the comparison between experimental and calculated spin-Hamiltonian param- 
eters allows us to attribute the spin $1 / 2$ signal to a localized radical on the DBTTF stack.

$E_{\mathrm{BG}}$ for the triplet state is in good agreement with the value obtained by elaborating the solid-state CV results. The work highlights DFTTF/F4TCNQ co-crystals as an interesting platform for spintronic devices because of the Mott electronic gap and the richness of spin degrees of freedom that could be controlled with temperature or external magnetic fields.

\section{ASSOCIATED CONTENT}

\section{SI Supporting Information}

The Supporting Information is available free of charge at https://pubs.acs.org/doi/10.1021/acs.jpcc.1c00020.

Estimation of HOMO and LUMO energy values from electrochemical results; EPR Q-band spectra and relevant DFT-calculated g-tensor; CV curves of F4TCNQ and DBTTF pristine and solid state; cocrystal CV curves as a function of the scan rate; analysis of the chemical stability (probed via UV-vis and CV measurements) of F4TCNQ and DBTTF in solution (PDF)

\section{AUTHOR INFORMATION}

\section{Corresponding Authors}

Enrico Da Como - Centre for Photonics and Photonic Materials (CPPM) and Department of Physics, University of Bath, Bath BA2 7AY, U.K.; 이이.org/0000-0002-5878371X; Email: edc25@bath.ac.uk

Agnese Giacomino - Department of Drug Science and Technology, University of Torino, Torino 10125, Italy; ○ orcid.org/0000-0001-6089-7751;

Email: agnese.giacomino@unito.it

\section{Authors}

Federica Solano - Department of Chemistry, University of Torino, Torino 10125, Italy

Paolo Inaudi - Department of Drug Science and Technology, University of Torino, Torino 10125, Italy

Mario Chiesa - Department of Chemistry, University of Torino, Torino 10125, Italy; (1) orcid.org/0000-0001-81288031

Gabriele Kociok-Köhn - Material and Chemical Characterization Facility $\left(M C^{2}\right)$, University of Bath, Bath BA2 7AY, U.K.; (1) orcid.org/0000-0002-7186-1399

Enrico Salvadori - Department of Chemistry, University of Torino, Torino 10125, Italy; o orcid.org/0000-0003-43949438

Davide Vanossi - DSCG, University of Modena and Reggio Emilia, 41125 Modena, Italy

Mery Malandrino - Department of Chemistry, University of Torino, Torino 10125, Italy

Raanan Carmieli - Department of Chemical Research Support, Weizmann Institute of Science, Rehovot 76100, Israel; 10 orcid.org/0000-0003-4418-916X

Claudio Fontanesi - DIEF, University of Modena and Reggio Emilia, 41125 Modena, Italy; 10 orcid.org/0000-00021183-2406

Complete contact information is available at:

https://pubs.acs.org/10.1021/acs.jpcc.1c00020

\section{Notes}

The authors declare no competing financial interest.

\section{REFERENCES}

(1) Sun, L.; Wang, Y.; Yang, F. X.; Zhang, X. T.; Hu, W. P. Cocrystal Engineering: A Collaborative Strategy toward Functional Materials. Adv. Mater. 2019, 31, 1902328.

(2) Goetz, K. P.; Vermeulen, D.; Payne, M. E.; Kloc, C.; McNeil, L. E.; Jurchescu, O. D. Charge-transfer complexes: new perspectives on an old class of compounds. J. Mater. Chem. C 2014, 2, 3065-3076.

(3) Saito, G.; Yoshida, Y. Development of conductive organic molecular assemblies: Organic metals, superconductors, and exotic functional materials. Bull. Chem. Soc. Jpn. 2007, 80, 1-137.

(4) Singleton, J. Why do physicists love charge-transfer salts? J. Solid State Chem. 2002, 168, 675-689.

(5) Ferraris, J.; Cowan, D. O.; Walatka, V.; Perlstein, J. H. Electrontransfer in a new highly conducting donor-acceptor complex. J. Am. Chem. Soc. 1973, 95, 948-949.

(6) Tomkiewicz, Y.; Taranko, A. R.; Torrance, J. B. Roles of donor and acceptor chains in metal-insulator transition in ttf-tcnq (tetrathiafulvalene tetracyanoquinodimethane). Phys. Rev. Lett. 1976, 36, 751-754.

(7) Torrance, J. B.; Vazquez, J. E.; Mayerle, J. J.; Lee, V. Y. Discovery of a neutral-to-ionic phase-transition in organic materials. Phys. Rev. Lett. 1981, 46, 253-257.

(8) Jiang, H.; Hu, P.; Ye, J.; Zhang, K. K.; Long, Y.; Hu, W.; Kloc, C. Tuning of the degree of charge transfer and the electronic properties in organic binary compounds by crystal engineering: a perspective. J. Mater. Chem. C 2018, 6, 1884-1902.

(9) Bray, J. W.; Interrante, L. V.; Jacobs, I. S.; Bonner, J. C. The Spin-Peierls Transition. In Extended Linear Chain Compounds: Volume 3; Miller, J. S., Ed.; Springer US: Boston, MA, 1983; pp 353-415.

(10) Miller, J. S. Extended Linear Chain Compounds; Plenum Press: New York and London, 1982.

(11) Zheng, Y.; Wudl, F. Organic spin transporting materials: present and future. J. Mater. Chem. A 2014, 2, 48-57.

(12) Kagawa, F.; Horiuchi, S.; Tokunaga, M.; Fujioka, J.; Tokura, Y. Ferroelectricity in a one-dimensional organic quantum magnet. Nat. Phys. 2010, 6, 169-172.

(13) D’Avino, G.; Souto, M.; Masino, M.; Fischer, J. K. H.; Ratera, I.; Fontrodona, X.; Giovannetti, G.; Verstraete, M. J.; Painelli, A.; Lunkenheimer, P.; et al. Conflicting evidence for ferroelectricity. Nature 2017, 547, E9-E10.

(14) Basura, V.; Beattie, P.; Holdcroft, S. Solid-state electrochemical oxygen reduction at Pt vertical bar Nafion (R) 117 and Pt vertical bar BAM3G (TM) 407 interfaces. J. Electroanal. Chem. 1998, 458, 1-5.

(15) Lufrano, F.; Staiti, P. Performance improvement of Nafion based solid state electrochemical supercapacitor. Electrochim. Acta 2004, 49, 2683-2689.

(16) Grygar, T.; Marken, F.; Schröder, U.; Scholz, F. Electrochemical analysis of solids. A review. Collect. Czech. Chem. Commun. 2002, 67, 163-208.

(17) Laudise, R. A.; Kloc, C.; Simpkins, P. G.; Siegrist, T. Physical vapor growth of organic semiconductors. J. Cryst. Growth 1998, 187, $449-454$.

(18) Henderson, J.; Masino, M.; Hatcher, L. E.; Kociok-Köhn, G.; Salzillo, T.; Brillante, A.; Raithby, P. R.; Girlando, A.; Da Como, E. New Polymorphs of Perylene:Tetracyanoquinodimethane Charge Transfer Cocrystals. Cryst. Growth Des. 2018, 18, 2003-2009.

(19) Sheldrick, G. M. Crystal structure refinement with SHELXL. Acta Crystallogr., Sect. C: Struct. Chem. 2015, 71, 3-8.

(20) Emge, T. J.; Bryden, W. A.; Wiygul, F. M.; Cowan, D. O.; Kistenmacher, T. J.; Bloch, A. N. Structure of an organic chargetransfer salt derived from dibenzotetrathiafulvalene and "tetrafluorotetracyanoquinodimethane (dbttf-tcnqf4) - observation of a hightemperature phase-transition. J. Chem. Phys. 1982, 77, 3188-3197.

(21) Hibbert, D. B.; Hamedelniel, A. E.; Sutcliffe, L. H. ESR and theoretical study of tetrathiofulvalene and dibenzotetrathiofulvalene and their radical cations. Magn. Reson. Chem. 1987, 25, 648-652.

(22) Metzger, R. M.; Heimer, N. E.; Gundel, D.; Sixl, H.; Harms, R. H.; Keller, H. J.; Nöthe, D.; Wehe, D. Crystal and molecular structure and ESR spectra of the 1:1 salt 5-(1-butyl)phenazinium (NBP)-2,2'- 
(2,3,5,6-tetrafluoro-2,5-cyclohexadiene- 1,4-diylidene)-bispropanedinitrile (TCNQF4). J. Chem. Phys. 1982, 77, 6203-6214.

(23) Le, T. H.; Nafady, A.; Qu, X.; Martin, L. L.; Bond, A. M. Detailed Electrochemical Analysis of the Redox Chemistry of Tetrafluorotetracyanoquinodimethane $\mathrm{TCNQF}(4)$, the Radical Anion TCNQF(4) (center dot-), and the Dianion TCNQF(4) (2-) in the Presence of Trifluoroacetic Acid. Anal. Chem. 2011, 83, 67316737.

(24) Li, J.; Duchemin, I.; Roscioni, O. M.; Friederich, P.; Anderson, M.; Da Como, E.; Kociok-Köhn, G.; Wenzel, W.; Zannoni, C.; Beljonne, D.; et al. Host dependence of the electron affinity of molecular dopants. Mater. Horiz. 2019, 6, 107-114.

(25) Meneghetti, M.; Pecile, C. Charge-transfer organic crystals: Molecular vibrations and spectroscopic effects of electron-molecular vibration coupling of the strong electron acceptor TCNQF4. J. Chem. Phys. 1986, 84, 4149-4162. 